\title{
Commentary: sex difference differences? A reply to Constantino
}

\author{
Daniel S. Messinger ${ }^{1 *}$, Gregory S. Young ${ }^{2}$, Sara Jane Webb ${ }^{3,4}$ (D), Sally Ozonoff², Susan E. Bryson ${ }^{5,6}$, Alice Carter ${ }^{7}$, \\ Leslie Carver ${ }^{8}$, Tony Charman ${ }^{9}$, Katarzyna Chawarska ${ }^{10}$, Suzanne Curtin ${ }^{11}$, Karen Dobkins ${ }^{8}$, Irva Hertz-Picciotto ${ }^{2}$, \\ Ted Hutman ${ }^{12}$, Jana M. Iverson ${ }^{13}$, Rebecca Landa ${ }^{14,15}$, Charles A. Nelson ${ }^{16,17,18}$, Wendy L. Stone ${ }^{4}$, \\ Helen Tager-Flusberg ${ }^{19}$ and Lonnie Zwaigenbaum ${ }^{20}$
}

\begin{abstract}
Messinger et al. found a 3.18 odds ratio of male to female ASD recurrence in 1241 prospectively followed high-risk (HR) siblings. Among high-risk siblings (with and without ASD), as well as among 583 low-risk controls, girls exhibited higher performance on the Mullen Scales of Early Learning, as well as lower restricted and repetitive behavior severity scores on the Autism Diagnostic Observation Schedule (ADOS) than boys. That is, female-favoring sex differences in developmental performance and autism traits were evident among low-risk and non-ASD high-risk children, as well as those with ASD. Constantino (Mol Autism) suggests that sex differences in categorical ASD outcomes in Messinger et al. should be understood as a female protective effect. We are receptive to Constantino's (Mol Autism) suggestion, and propose that quantitative sex differences in autism-related features are keys to understanding this female protective effect.
\end{abstract}

Keywords: Female protective effect, Sex differences, High-risk siblings, Autism spectrum disorder

\section{Background}

Prospective studies of the high-risk siblings of children with ASD offer an opportunity to examine both sex differences in ASD occurrence and sex differences in ASD traits and related cognitive characteristics. Messinger et al. [1] reported on a large sample (1241 high-risk siblings and 583 low-risk children) recruited at a mean age of 7.25 months, whose cognitive/developmental performance on the Mullen Scales of Early Learning (MSEL) and autism severity scores on the Autism Diagnostic Observation Schedule (ADOS) were assessed at 24 and 36 months. ASD outcome (assessed at 36 months) required both a clinical best estimate diagnosis of ASD as well as an ADOS severity score $\geq 4$.

The female protective effect may be operationalized with respect to the Carter effect, which holds that siblings of female ASD probands will evidence greater ASD affectation (more severe autism traits) than siblings of male probands [2, 3]. This argument has been buttressed by findings of increased genetic liability (e.g., deleterious copy number variants and single-nucleotide variants) in

\footnotetext{
* Correspondence: dmessinger@miami.edu

'University of Miami, Coral Gables, USA

Full list of author information is available at the end of the article
}

female ASD probands [4]. However, Messinger et al. found that the high-risk siblings of female ASD probands did not differ significantly in ASD outcome, Mullen performance, or severity scores from siblings of male ASD probands. In other words, proband sex was not consequential for the younger high-risk sibling's outcome.

Constantino suggests that sex differences in categorical ASD clinical outcomes among the high-risk siblings themselves should be understood as a female protective effect [5]. We are open to this formulation. Quantifying sex differences in categorical ASD outcome is important to understanding the female protective effect. The male to female odds ratio for ASD outcome in Messinger et al. was 3.18, similar to that reported in population-based studies of affected children [6,7]. The accompanying commentary ([5], see Fig. 1) noted that there were over three times more ASD outcomes among male (193) than female (59) HR siblings. This is correct, but does not reflect the higher absolute number of male HR siblings. The proportion of HR siblings with ASD outcomes was $.27(193 / 714)$ for males and .11 (59/527) for females (see Fig. 1).

Messinger et al. found that the under-representation of female categorical ASD outcomes was accompanied 


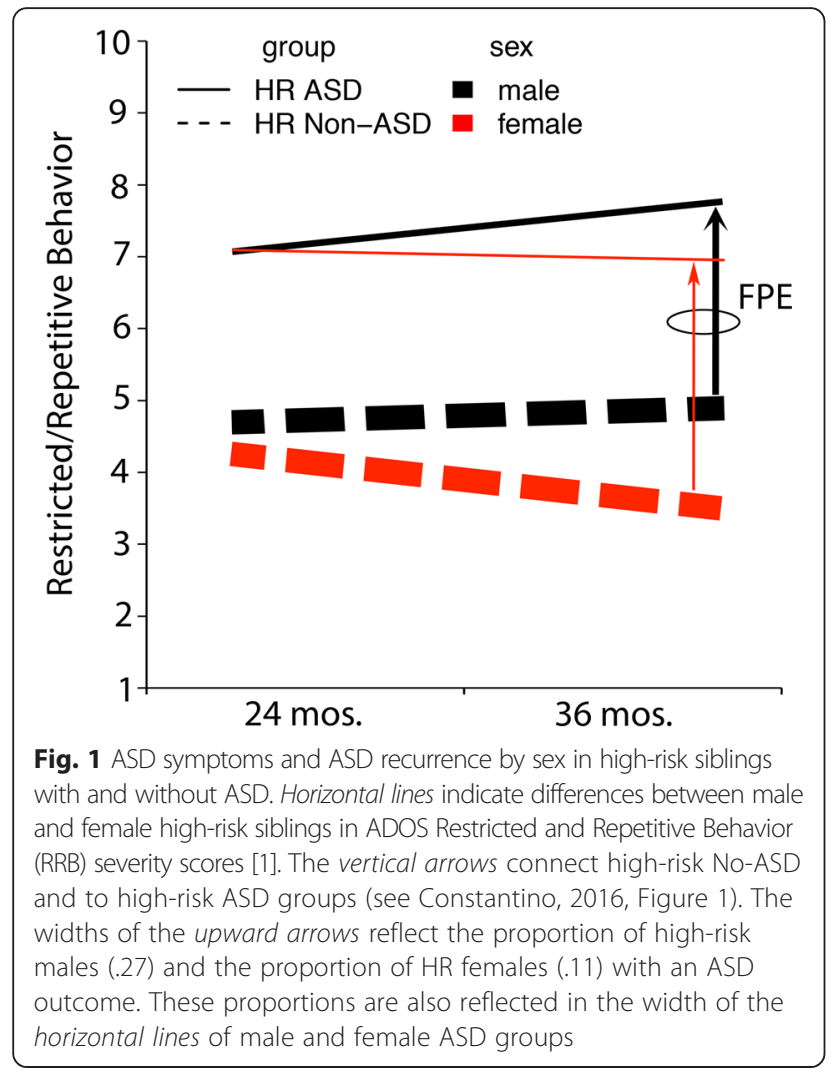

by female-favoring sex differences in quantitatively distributed ASD-relevant behaviors [1]. Figure 1 displays restricted and repetitive behavior severity scores from the ADOS, which provide a context for visualizing differences among high-risk siblings in categorical ASD outcomes. Among HR-ASD and HR No-ASD siblings, as well as among LR infants, girls exhibited both higher levels of cognitive/developmental performance and lower levels of restricted and repetitive behaviors than boys. Sex differences, while smaller than the differences between high-risk siblings with and without ASD, were characterized by medium to large effect sizes. An absence of group by sex interaction effects indicated that sex differences in children with ASD were not autism specific; they were also evident in high-risk siblings without ASD and low-risk children.

Overall decrements in quantitative indices of autistic traits among females are common in high-risk siblings with and without ASD [8-11] as well as in the general population [12]. It is likely that population-level reductions in ASD-relevant traits among females lower the propensity for ASD occurrence among females. While certainly not a full explanation for higher rates of ASD among males, sex differences in ASD-relevant traits are pertinent to and, in fact, may be manifestations of a female protective effect.

\section{Conclusions}

The relationship between sex differences in quantitative autistic traits and categorical ASD outcomes involves examination of commonalities and differences in the presentation and causes of ASD in males and females [13-15]. While quantitative and categorical outcomes were not independent in Messinger et al., the large scale prospective results indicate both a robust female advantage in most but not all autism-related traits, and a threefold reduction in female ASD outcomes. Ultimately, a female protective effect manifested in a lower rate of categorical ASD outcomes must be informed by a thorough understanding of female-favoring sex differences in quantitative ASD traits.

\section{Abbreviations}

ADOS, autism diagnostic observation schedule; ASD, autism spectrum disorder; BSRC, Baby Siblings Research Consortium; HR No-ASD, high-risk group without ASD outcomes; HR, high-risk group; HR-ASD, high-risk group with ASD outcomes; LR, low risk group; MSEL, Mullen scales of early learning; RRB, Restricted and Repetitive Behavior Domain Calibrated Severity Score from the Autism Diagnostic Observation Schedule

\section{Acknowledgements}

Not applicable.

\section{Funding}

Autism Speaks provided support for the creation of the Baby Siblings Research Consortium (BSRC) Database. Data collection, analyses, and manuscript preparation were supported by the National Institutes of Health (C.A.N. R01DC010290; D.M. R01HD057284; D.M. R01GM105004; H.T.F. R01DC010290; H.T.F.R21DC008637; K.C. R01MH087554; K.C. PO1HD003008, Project 1; J.M.I. R01HD054979; R.L. R01MH059630; S.O R01MH068398; S.JW. P5OHD055782; S.J.W. R01HD064820; T.H. K01MH096961; T.H. U54 MH068172; T. H. P50 HD55784; W.L.S R01HD057284); the Canadian Institutes of Health Research (S.B., L.Z.); the UK Medical Research Council (T.C.); NeuroDevNet (S.B., L.Z.), Autism Speaks Canada (S.B., L.Z.); Autism Speaks [U.S.] Pilot Grant (H.T.F., J.M.I.); Autistica (T.C.); the FAR Fund (K.C.); and the Simons Foundation (C.A.N.).

Availability of data and materials Not applicable.

\section{Authors' contributions}

DSM drafted the manuscript and GSY created Fig. 1. SJW, SO, SB, AC, LC, TC, $K C, S C, K D, I H-P, T H, J M I, R L, C A N, W L S, H T-F$, and $L Z$ edited or provided feedback on the manuscript. All authors read and approved the manuscript.

\section{Competing interests}

Dr. Zwaigenbaum receives operating funds but not honoraria from SynapDx. Dr. Charman receives royalties from Guilford Press and Sage Publications. Drs. Bryson, Carter, Carver, Chawarska, Dobkins, Iverson, Landa, Hutman, Messinger, Nelson, Ozonoff, Stone, Tager-Flusberg, Webb, and Young declare that they have no competing interests.

Consent for publication

Not applicable.

Ethics approval and consent to participate Not applicable.

\section{Author details}

${ }^{1}$ University of Miami, Coral Gables, USA. ²University of California, Davis, Davis, USA. ${ }^{3}$ Seattle Children's Research Institute, Seattle, USA. ${ }^{4}$ University of Washington, Seattle, USA. ${ }^{5}$ zaak Walton Killam Health Centre, Halifax, Canada. ${ }^{6}$ Dalhousie University, Halifax, Canada. ${ }^{7}$ University of Massachusetts, Boston, Boston, USA. ${ }^{8}$ University of California, San Diego, La Jolla, USA. 
${ }^{9}$ King's College London, London, UK. ${ }^{10}$ Yale University School of Medicine, New Haven, USA. ${ }^{11}$ University of Calgary, Calgary, Canada. ${ }^{12}$ University of California, Los Angeles, Los Angeles, USA. ${ }^{13}$ University of Pittsburgh, Pittsburgh, USA. ${ }^{14}$ Kennedy Krieger Institute, Baltimore, USA. ${ }^{15}$ John Hopkins School of Medicine, Baltimore, USA. ${ }^{16}$ Harvard Medical School, Boston, USA.

${ }^{17}$ Harvard Graduate School of Education, Cambridge, USA. ${ }^{18}$ Boston

Children's Hospital, Boston, USA. ${ }^{19}$ Boston University, Boston, USA.

${ }^{20}$ University of Alberta, Edmonton, Canada.

Received: 18 May 2016 Accepted: 6 June 2016

\section{Published online: 29 June 2016}

\section{References}

1. Messinger DS et al. Early sex differences are not autism-specific: a Baby Siblings Research Consortium (BSRC) study. Mol Autism. 2015;6:32.

2. Robinson $E B$ et al. Examining and interpreting the female protective effect against autistic behavior. Proc Natl Acad Sci U S A. 2013;110(13):5258-62

3. Jacquemont $\mathrm{S}$ et al. A higher mutational burden in females supports a "female protective model" in neurodevelopmental disorders. Am J Hum Genet. 2014;94(3):415-25.

4. Jacquemont S, Coe BP, Hersch M, Duyzend MH, Krumm N, Bergmann S, Eichler EE (2014). A higher mutational burden in females supports a "female protective model" in neurodevelopmental disorders. Am J Hum Genet. 2014;94(3):415-425. doi: 10.1016/j.ajhg.2014.02.001

5. Constantino JN. Data from the Baby Siblings Research Consortium confirm and specify the nature of the female protective effect in autism: a commentary on Messinger et al. Mol Autism. 2016. In press.

6. Kim YS et al. Prevalence of autism spectrum disorders in a total population sample. Am J Psychiatry. 2011;168(9):904-12.

7. Idring $\mathrm{S}$ et al. Autism spectrum disorders in the Stockholm youth cohort: design, prevalence and validity. PLoS ONE. 2012;7(7):e41280.

8. Virkud $Y$ et al. Familial aggregation of quantitative autistic traits in multiplex versus simplex autism. Am J Med Genet B Neuropsychiatr Genet. 2009;150b(3):328-34.

9. Hartley SL, Sikora DM. Sex differences in autism spectrum disorder: an examination of developmental functioning, autistic symptoms, and coexisting behavior problems in toddlers. J Autism Dev Disord. 2009;39(12):1715-22.

10. Szatmari $P$ et al. Sex differences in repetitive stereotyped behaviors in autism: implications for genetic liability. Am J Med Genet B Neuropsychiatr Genet. 2012;159b(1):5-12.

11. Frazier TW, Georgiades S, Bishop SL, Hardan AY. (2014). Behavioral and cognitive characteristics of females and males with autism in the Simons Simplex Collection. J Am Acad Child Adolesc Psychiatry. 2014;53(3):329-340. e321-323. doi: 10.1016/j.jaac.2013.12.004

12. Constantino JN, Todd RD. Intergenerational transmission of subthreshold autistic traits in the general population. Biol Psychiatry. 2005;57(6):655-60.

13. Russell G, Steer C, Golding J. Social and demographic factors that influence the diagnosis of autistic spectrum disorders. Soc Psychiatry Psychiatr Epidemiol. 2011;46(12):1283-93.

14. Lai $M$ et al. Sex/gender differences and autism: setting the scene for future research. J Am Acad Child Adolesc Psychiatry. 2015;54(1):11-24.

15. Robinson EB et al. Genetic risk for autism spectrum disorders and neuropsychiatric variation in the general population. Nat Genet. 2016. Advance online publication.

\section{Submit your next manuscript to BioMed Central and we will help you at every step:}

- We accept pre-submission inquiries

- Our selector tool helps you to find the most relevant journal

- We provide round the clock customer support

- Convenient online submission

- Thorough peer review

- Inclusion in PubMed and all major indexing services

- Maximum visibility for your research

Submit your manuscript at www.biomedcentral.com/submit
Biomed Central 